\title{
TITLE:
}

\section{Flat manifold leptogenesis in the supersymmetric standard model}

$\operatorname{AUTHOR}(S)$ :

Senami, M; Yamamoto, $\mathrm{K}$

CITATION:

Senami, M ... [et al]. Flat manifold leptogenesis in the supersymmetric standard model. PHYSICAL REVIEW D 2002, 66(3): 035006.

ISSUE DATE:

2002-08-01

URL:

http://hdl.handle.net/2433/39895

RIGHT:

Copyright 2002 American Physical Society 
PHYSICAL REVIEW D 66, 035006 (2002)

\title{
Flat manifold leptogenesis in the supersymmetric standard model
}

\author{
Masato Senami* and Katsuji Yamamoto ${ }^{\dagger}$ \\ Department of Nuclear Engineering, Kyoto University, Kyoto 606-8501, Japan
}

(Received 6 May 2002; published 23 August 2002)

Flat manifold leptogenesis in the manner of Affleck and Dine is investigated with the slepton and Higgs fields, $\widetilde{L}, H_{u}, H_{d}$, in the supersymmetric standard model. The multidimensional motion of these scalar fields is realized in the case that the $\widetilde{L} H_{u}$ and $H_{u} H_{d}$ directions are comparably flat with the relevant nonrenormalizable superpotential terms. Soon after inflation, lepton number asymmetry appears to fluctuate due to this multidimensional motion involving certain $C P$ violating phases. Then, it is fixed to some significant nonzero value for the successful baryogenesis when the scalar fields begin to oscillate with rotating phases driven by the quartic coupling from the superpotential term $\bar{h}_{e} L H_{d} e^{c}$ with $\bar{h}_{e} \sim 10^{-5}-10^{-3}$. The Hubble parameter $H_{\mathrm{osc}}$ at this epoch for the completion of leptogenesis is much larger than the gravitino mass $m_{3 / 2} \sim 10^{3} \mathrm{GeV}$. The thermal terms may even play a cooperative role in this scenario of early leptogenesis. The lightest neutrino mass can be $m_{\nu_{1}} \sim 10^{-4} \mathrm{eV}$, if the reheating temperature is allowed to be $T_{R} \sim 10^{10} \mathrm{GeV}$.

DOI: 10.1103/PhysRevD.66.035006

PACS number(s): $12.60 . J v$

\section{INTRODUCTION}

The Affleck-Dine (AD) mechanism [1,2] is well known as one of the promising candidates to explain the long-standing cosmological problem, baryogenesis, in supersymmetric models. Particularly, the $\widetilde{L} H_{u}$ flat direction has been investigated extensively for leptogenesis in the supersymmetric standard model, where the generated lepton number asymmetry is converted with a significant fraction to the baryon number asymmetry through the anomalous electroweak process [2-5]. In this $\widetilde{L} H_{u}$ case, there appears an interesting relation between the baryon number asymmetry and the neutrino masses through the superpotential term $\left(L H_{u}\right)\left(L H_{u}\right)$. It should be noticed here that the $\left(H_{u} H_{d}\right)\left(H_{u} H_{d}\right)$ term is implicitly assumed to be larger than the $\left(L H_{u}\right)\left(L H_{u}\right)$ term. Otherwise, the $H_{u} H_{d}$ would be the flattest direction without producing the lepton number asymmetry.

In this paper, we reexamine leptogenesis in the manner of Affleck and Dine in the supersymmetric standard model by considering the new possibility that a flat manifold is formed for the $\widetilde{L}, H_{u}$, and $H_{d}$ fields with comparable $\left(L H_{u}\right)\left(L H_{u}\right)$ and $\left(H_{u} H_{d}\right)\left(H_{u} H_{d}\right)$ terms. This comparability seems to be plausible if these nonrenormalizable terms stem from the physics at the Planck scale. It has been observed recently in an extension of the supersymmetric standard model with a Higgs triplet that the multidimensional motion can really be realized for the scalar fields on a flat manifold [6]. In the multidimensional motion on the flat manifold, nonconservation of certain particle numbers with effective $C P$ violation may be available from some potential terms with different dependences on the scalar field phases. Then, soon after inflation the fluctuating motion of the scalar fields appears due to the effects of these potential terms, and the particle number asymmetries such as the $B-L$ asymmetry are generated varying in time. We will show in the text that this sort of

\footnotetext{
*Email address: senami@nucleng.kyoto-u.ac.jp

†Email address: yamamoto@nucleng.kyoto-u.ac.jp
}

phenomenon indeed occurs for the flat manifold leptogenesis with the scalar fields $\widetilde{L}, H_{u}$, and $H_{d}$ in the supersymmetric standard model. It is the novel point in the present scenario that the lepton number asymmetry fluctuating after the inflation is fixed to some significant nonzero value due to the effect of the superpotential term $\bar{h}_{e} L H_{d} e^{c}$. While the quartic term $\bar{h}_{e}^{2}\left|\widetilde{L} H_{d}\right|^{2}$ is safely small during the inflation with $\bar{h}_{e}$ $\sim 10^{-5}-10^{-3}$, it in turn provides the driving force for the scalar fields to oscillate with rotating phases at some epoch after the inflation. The Hubble parameter $H_{\text {osc }}$ at this epoch of the onset of oscillation by the $\bar{h}_{e}$ quartic term can be much larger than the gravitino mass $m_{3 / 2} \sim 10^{3} \mathrm{GeV}$. The leptogenesis is completed in this quite early epoch with $H \sim H_{\text {osc }}$, which may even be before the thermal terms $[4,5,7]$ become significant. Hence this flat manifold leptogenesis is not restricted by the physics at the electroweak scale such as the low-energy supersymmetry breaking terms. This is in salient contrast to the conventional flat direction leptogenesis.

This paper is organized as follows. In Sec. II, the relevant part of the supersymmetric standard model is presented for the flat manifold leptogenesis. In Sec. III, the mechanism of flat manifold leptogenesis is described following the respective epochs starting with the inflation, and the resultant lepton number asymmetry is estimated specifically related to the lightest neutrino mass. In Sec. IV, the thermal effects are discussed to show that they do not alter essentially the present scenario for leptogenesis. In Sec. V, detailed numerical calculations are made to confirm the multidimensional motion of the scalar fields, and the reasonable parameter range for the sufficient leptogenesis is identified. Section VI is finally devoted to the conclusion of this investigation for the flat manifold leptogenesis.

\section{MODEL}

We investigate the supersymmetric standard model including the nonrenormalizable superpotential terms, 


$$
W_{\text {non }}=\frac{\lambda_{E_{i}}}{2 M}\left(L_{i} H_{u}\right)\left(L_{i} H_{u}\right)+\frac{\lambda_{H}}{2 M}\left(H_{u} H_{d}\right)\left(H_{u} H_{d}\right),
$$

where $M$ represents some very large mass scale such as the Planck scale, and the suitable basis is chosen for the relevant fields to give the positive and diagonal $\lambda_{E_{i}}$ and positive $\lambda_{H}$. The $R$-parity violating terms $\left(L_{i} H_{u}\right)\left(H_{u} H_{d}\right)$ are not included for simplicity. These terms do not alter the present leptogenesis scenario if they are not significantly large compared to $W_{\text {non }}$. We here assume the condition on the terms in $W_{\text {non }}$,

$$
\lambda_{H} \sim \lambda_{E_{1}} \ll \lambda_{E_{2}}, \lambda_{E_{3}} .
$$

Then, the flat manifold for leptogenesis is formed with the $\widetilde{L}_{1}, H_{u}$, and $H_{d}$ fields. The lepton doublet $L_{1}$ includes the lightest neutrino $\nu_{1}$ with a mass

$$
m_{\nu_{1}}=\lambda_{E_{1}} \frac{\left\langle H_{u}\right\rangle^{2}}{M} \sim 10^{-6} \mathrm{eV}\left(\frac{10^{19} \mathrm{GeV}}{M / \lambda_{E_{1}}}\right)
$$

where $\sqrt{\left\langle H_{u}\right\rangle^{2}+\left\langle H_{d}\right\rangle^{2}}=174 \mathrm{GeV}$ with $\left\langle H_{u}\right\rangle /\left\langle H_{d}\right\rangle \equiv \tan \beta$. The comparability of $\lambda_{t_{1}}$ and $\lambda_{H}$ would be understood by considering that these nonrenormalizable terms stem from the physics at the Planck scale. On the other hand, as indicated from the solar and atmospheric neutrino puzzles, the other two neutrino masses are probably around $10^{-2} \mathrm{eV}$, apparently requiring $\lambda_{E_{2}}, \lambda_{E_{3}} \sim 10^{4}\left(M / 10^{19} \mathrm{GeV}\right)$. Such large couplings may be obtained effectively as $\lambda_{E_{2}}, \lambda_{E_{3}}$ $\sim \lambda_{E_{1}}\left(M / M_{\nu^{c}}\right)$ by the so-called seesaw mechanism with heavy right-handed neutrinos at an intermediate scale $M_{\nu^{c}}$ $\ll M[8]$. The see-saw contribution to $\lambda_{\iota_{1}} / M$ should, however, be suppressed sufficiently for the successful leptogenesis.

The superpotential terms relevant for the flat manifold leptogenesis are given by

$$
W=\left(h_{e}\right)_{i j} L_{i} H_{d} e_{j}^{c}+\mu H_{u} H_{d}+W_{\text {non }} .
$$

The $F$ terms except for the contributions of $W_{\text {non }}$ are then calculated as

$$
\begin{gathered}
F_{L_{i}}=\left(h_{e}\right)_{i j} H_{d} e_{j}^{c}, \quad F_{e_{j}^{c}}=\left(h_{e}\right)_{i j} L_{i} H_{d}, \\
F_{H_{u}}=\mu H_{d}, \quad F_{H_{d}}=\left(h_{e}\right)_{i j} L_{i} e_{j}^{c}+\mu H_{u} .
\end{gathered}
$$

Among the slepton fields, only the sneutrino $\tilde{\nu}_{1}$ in $\widetilde{L}_{1}$ associated with the lightest neutrino is considered to develop a very large coherent field value during the inflation, according to condition (2). Then, it is usually supposed that the $\tilde{\nu}_{1}$ and $H_{d}^{0}$ are incompatible for the flat directions due to the $F_{e^{c}}$ terms. However, this is not necessarily the case in the present scenario. In fact, the $F_{e^{c}}$ terms provide the quartic term

$$
\bar{h}_{e}^{2}\left|\widetilde{L}_{1} H_{d}\right|^{2}
$$

with

$$
\bar{h}_{e}^{2} \equiv \sum_{j}\left|\left(h_{e}\right)_{1 j}\right|^{2}
$$

The effective coupling is estimated as

$$
\bar{h}_{e} \simeq \frac{m_{e}+\sin \theta_{12} m_{\mu}}{\left\langle H_{d}\right\rangle} \sim \begin{cases}10^{-5} & \left(\theta_{12} \lesssim 10^{-2}\right) \\ 10^{-3} & \left(\theta_{12} \sim 1\right)\end{cases}
$$

where $\theta_{12}$ represents the $\nu_{e}-\nu_{\mu}$ mixing, and the effect of the $\nu_{e}-\nu_{\tau}$ mixing is assumed to be small enough. As explained in Sec. III, while this quartic term is safely small during the inflation with $\bar{h}_{e} \sim 10^{-5}-10^{-3}$, it in turn provides the driving force for the scalar fields to oscillate with rotating phases at some epoch after the inflation. This is one of the essential points in the present scenario for the flat manifold leptogenesis.

According to the above arguments, the flat manifold is specified by the $D$-flat condition for the $\mathrm{SU}(2)_{L} \times \mathrm{U}(1)_{Y}$ gauge interactions,

$$
\left|\tilde{\nu}_{1}\right|^{2}-\left|H_{u}^{0}\right|^{2}+\left|H_{d}^{0}\right|^{2}=0
$$

and the other fields are vanishing. Henceforth, we adopt for simplicity the notation $\widetilde{L} \equiv \widetilde{\nu}_{1}, H_{u} \equiv H_{u}^{0}$, and $H_{d} \equiv H_{d}^{0}$, suppressing the lepton generation indices. Including the contributions of $W_{\text {non }}$, this manifold is flat enough for both the $\widetilde{L} H_{u}$ and $H_{u} H_{d}$ directions in the case that $\lambda_{t}$ and $\lambda_{H}$ are comparable,

$$
0.3 \lesssim \lambda_{t} / \lambda_{H} \lesssim 3,
$$

as observed in Ref. [6].

\section{FLAT MANIFOLD LEPTOGENESIS}

We here describe the mechanism of leptogenesis on the flat manifold consisting of the scalar fields $\widetilde{L}, H_{u}, H_{d}$, say $A D$-flatons. (The scalar fields associated with the flat potential are intrinsic in supersymmetric models, which are named flatons [9]. Here, we consider such fields as participants in leptogenesis/baryogenesis in the manner of Affleck and Dine.) The scalar potential for the AD-flatons $\phi_{a}$ $=\widetilde{L}, H_{u}, H_{d}$ may be presented as

$$
V=V_{\text {high }}+\bar{h}_{e}^{2}\left|\tilde{L} H_{d}\right|^{2}+V_{\text {low }} .
$$

These three parts become dominant in the high, middle, and low energy scales, respectively. The evolution of the ADflaton fields is traced in the corresponding epochs starting with the inflation. The high-energy part is provided from the superpotential $W_{\text {non }}$ with the mass scale $M$ and the corresponding soft supersymmetry breaking with the Hubble parameter $H$ : 


$$
\begin{aligned}
V_{\text {high }}= & -\sum_{a} c_{a} H^{2}\left|\phi_{a}\right|^{2}+\left|\lambda_{E} \frac{\widetilde{L}\left(\widetilde{L} H_{u}\right)}{M}+\lambda_{H} \frac{H_{d}\left(H_{u} H_{d}\right)}{M}\right|^{2} \\
& +\left|\lambda_{H} \frac{H_{u}\left(H_{u} H_{d}\right)}{M}\right|^{2}+\left|\lambda_{E} \frac{H_{u}\left(\widetilde{L} H_{u}\right)}{M}\right|^{2} \\
& +\left[\frac{\lambda_{E}}{2 M} a_{E} H\left(\widetilde{L} H_{u}\right)\left(\widetilde{L} H_{u}\right)+\text { H.c. }\right] \\
& +\left[\frac{\lambda_{H}}{2 M} a_{H} H\left(H_{u} H_{d}\right)\left(H_{u} H_{d}\right)+\text { H.c. }\right] \\
& +\left(g^{2}+g^{\prime 2}\right)\left(|\widetilde{L}|^{2}-\left|H_{u}\right|^{2}+\left|H_{d}\right|^{2}\right)^{2} .
\end{aligned}
$$

The last term with the gauge couplings $g$ and $g^{\prime}$ of the $\mathrm{SU}(2)_{L}$ and $\mathrm{U}(1)_{Y}$, respectively, is also included here to realize the $D$-flat condition (9) for the very large $|\widetilde{L}|,\left|H_{u}\right|$, and $\left|H_{d}\right|$. The low-energy part $V_{\text {low }}$ includes the remaining terms, which are related to the parameters $m_{3 / 2},|\mu|$ $\sim 10^{3} \mathrm{GeV}$. It will be clarified in the following that the quartic term $\bar{h}_{e}^{2}\left|\widetilde{L} H_{d}\right|^{2}$ plays a crucial role to complete the flat manifold leptogenesis. On the other hand, the low-energy part $V_{\text {low }}$ is actually irrelevant for the leptogenesis. This is in salient contrast to the conventional flat direction AffleckDine mechanism. In this section we do not either consider explicitly the thermal terms [4,5,7]. It will be seen in Secs. IV and V that the thermal terms do not alter essentially the present scenario of flat manifold leptogenesis. They may even play a cooperative role for leptogenesis.

\section{A. $\boldsymbol{H}=\boldsymbol{H}_{\text {inf }}$}

During the inflation the Hubble parameter takes almost a constant value $H_{\text {inf }}$, which is typically $10^{14} \mathrm{GeV}$ or so. In this epoch, the AD-flaton fields quickly settle into one of the minima of the scalar potential (11) with $H=H_{\text {inf }}$. The potential minima is almost determined by the high-energy part $V_{\text {high }}$ as

$$
\phi_{a}^{(0)}=\mathrm{e}^{i \theta_{a}^{(0)}} r_{a}^{(0)} \sqrt{H_{\mathrm{inf}}(M / \lambda)},
$$

with $\lambda$ representing the mean value of $\lambda_{E}$ and $\lambda_{H}$. The generation of these nontrivial minima (13) with $r_{a}^{(0)} \sim 0.1-1$ far apart from the origin on the multidimensional flat manifold depends rather complicatedly on the parameters $c_{a}, \lambda_{E}, \lambda_{H}$, $a_{E}$, and $a_{H}$ [6]. As usually considered, at least one of $c_{a}$ 's should be positive so that the origin stays unstable in the inflation epoch. It is also essential for the flat manifold formation that $\lambda_{E}$ and $\lambda_{H}$ are comparable, as given in Eq. (10). Otherwise, the potential minima would be developed along either the $\widetilde{L} H_{u}$ direction or the $H_{u} H_{d}$ direction.

By comparing $V_{\text {high }} \sim H_{\text {inf }}^{3}(M / \lambda) \quad$ and $\quad \bar{h}_{e}^{2}\left|\widetilde{L} H_{d}\right|^{2}$ $\sim \bar{h}_{e}^{2} H_{\mathrm{inf}}^{2}(M / \lambda)^{2}$, the following condition is expected to be satisfied for the flatness of the $\widetilde{L}-H_{u}-H_{d}$ manifold during the inflation:

$$
H_{\mathrm{inf}}>H_{\mathrm{osc}} \equiv \bar{h}_{e}^{2}(M / \lambda) .
$$

The critical value $H_{\mathrm{osc}}$ of the Hubble parameter for which the $\bar{h}_{e}$ quartic term becomes comparable to $V_{\text {high }}$ may be estimated in terms of $m_{\nu_{1}}$ with Eqs. (3) and (8):

$$
H_{\mathrm{osc}} \sim 10^{9} \mathrm{GeV}\left(\frac{\bar{h}_{e}}{10^{-5}}\right)^{2}\left(\frac{10^{-6} \mathrm{eV}}{m_{\nu_{1}}}\right) .
$$

Hence condition (14) can be satisfied readily since $H_{\text {inf }}$ is typically $10^{14} \mathrm{GeV}$ or so. Here, it may be noticed that if $\bar{h}_{e}$ $\sim 10^{-3}$ with large $\nu_{e}-\nu_{\mu}$ mixing, $H_{\text {osc }}$ could be comparable to $H_{\text {inf }}$. Remarkably, even in such an extreme case with $H_{\text {osc }} \sim H_{\text {inf }}$ the successful leptogenesis can be realized, as confirmed by the numerical calculations in Sec. V.

$$
\text { B. } \boldsymbol{H}_{\text {osc }}<\boldsymbol{H}<\boldsymbol{H}_{\text {inf }}
$$

After the inflation the inflaton oscillates coherently and it dominates the energy density of the universe. In this epoch with $H>H_{\text {osc }}$, the high-energy part $V_{\text {high }}$ is still dominant, and the evolution of the AD-flaton fields is essentially the same as in the case of the model with triplet Higgs [6]. We recapitulate the main results with suitable changes of notation, showing especially the fluctuating behavior of the lepton number asymmetry after the inflation.

The AD-flaton fields are moving toward the origin with the initial conditions at $t=t_{0} \sim H_{\text {inf }}^{-1}$ after the inflation,

$$
\phi_{a}\left(t_{0}\right)=\phi_{a}^{(0)}, \quad \dot{\phi}_{a}\left(t_{0}\right)=0 .
$$

The evolution of the AD-flaton fields is governed by the equations of motion,

$$
\ddot{\phi}_{a}+3 H \dot{\phi}_{a}+\frac{\partial V}{\partial \phi_{a}^{*}}=0
$$

The Hubble parameter varies in time as $H=(2 / 3) t^{-1}$ in the matter-dominated universe. The AD-flaton fields may be represented suitably in terms of the dimensionless fields $\chi_{a}$ [2] as

$$
\phi_{a}=\chi_{a} \sqrt{H(M / \lambda)} \equiv \mathrm{e}^{i \theta_{a}} r_{a} \sqrt{H(M / \lambda)} .
$$

Then, the equations of motion (17) are rewritten with $z$ $=\ln \left(t / t_{0}\right)$ as

$$
\frac{d^{2} \chi_{a}}{d z^{2}}+\frac{\partial U}{\partial \chi_{a}^{*}}=0
$$

and the initial conditions from Eq. (16) are given as

$$
\chi_{a}(0)=\mathrm{e}^{i \theta_{a}^{(0)}} r_{a}^{(0)}, \quad \frac{d \chi_{a}}{d z}(0)=\frac{1}{2} \chi_{a}(0) .
$$

It should be noticed in Eq. (19) that the first-order $z$ derivative is absent due to the parametrization of $\phi_{a}$ in Eq. (18). The dimensionless effective potential is given dominantly by 


$$
U\left(\chi_{a}\right) \simeq \frac{4}{9 H^{3}(M / \lambda)} V_{\mathrm{high}}-\frac{1}{4}\left|\chi_{a}\right|^{2}+\frac{4}{9}\left(H_{\mathrm{osc}} / H\right)\left|\chi_{\tilde{L}} \chi_{H_{d}}\right|^{2} .
$$

The second term is due to the time variation of the factor $\sqrt{H(M / \lambda)}$ in Eq. (18), which apparently provides the change of the mass terms in $U\left(\chi_{a}\right)$,

$$
c_{a} \rightarrow c_{a}+\frac{9}{16} .
$$

The third contribution from the $\bar{h}_{e}$ quartic term is small enough in this epoch with $H>H_{\text {osc }}$, while it will in turn play a crucial role in the next epoch. The low-energy part $V_{\text {low }}$ is clearly negligible for $H_{\mathrm{osc}} \gg m_{3 / 2},|\mu|$.

The behavior of the AD-flaton phases $\theta_{a}$ is described in this epoch as follows. The initial conditions at $t=t_{0}(z=0)$ are given from Eq. (20) as

$$
\theta_{a}(0)=\theta_{a}^{(0)}, \quad \frac{d \theta_{a}}{d z}(0)=0 .
$$

On the other hand, the asymptotic trajectory of the AD-flaton fields is found by the conditions $\partial U / \partial \chi_{a}^{*}=0$ in this epoch with the small enough $\bar{h}_{e}$ quartic term as

$$
\theta_{a}=\theta_{a}^{(1)} .
$$

It is remarkable in the multidimensional motion of the $\mathrm{AD}$ flaton fields with $\lambda_{E} \sim \lambda_{H}$ that the direction of this trajectory is somewhat different from the initial direction, i.e.,

$$
\theta_{a}^{(1)} \neq \theta_{a}^{(0)} .
$$

This is because the apparent change of the mass terms in Eq. (22) due to the redshift induces the new balance among the $\lambda_{E}-\lambda_{H}$ cross term, $a_{E}$ term, and $a_{H}$ term, in $U\left(\chi_{a}\right)$, which have different dependences on $\theta_{a}$. [If the fine-tuning is made as $\arg \left(a_{E}\right)-\arg \left(a_{H}\right)=\pi \bmod 2 \pi$, the initial balance is maintained independently of $\left|\chi_{a}\right|$ so as to realize $\theta_{a}^{(0)}$ $=\theta_{a}^{(1)}$.] Without the $d \chi_{a} / d z$ (friction) term in Eq. (19), the AD-flaton phases $\theta_{a}$ slowly fluctuate around $\theta_{a}^{(1)}$ starting from $\theta_{a}^{(0)}$ as a function of $z=\ln \left(t / t_{0}\right)$ in the epoch $H_{\text {inf }}^{-1} \sim t_{0}$ $\leqslant t<H_{\mathrm{osc}}^{-1}$. That is, in the motion on the multidimensional flat manifold the AD-flaton fields no longer track exactly behind the decreasing instantaneous minimum of the scalar potential. This is in salient contrast to the conventional Affleck-Dine mechanism on the one-dimensional flat direction, where the AD-flaton phase is fixed for a long time until the low-energy supersymmetry breaking terms become important.

In this way, through this fluctuating motion of the (almost) homogeneous coherent AD-flaton fields $\phi_{a}(t)$, the particle number asymmetries are generated soon after the inflation as

$$
\Delta n_{a} \equiv n_{a}-\bar{n}_{a}=i\left(\phi_{a}^{*} \dot{\phi}_{a}-\dot{\phi}_{a}^{*} \phi_{a}\right) .
$$

The fractions of the respective asymmetries are also calculated by considering the redshift in the matter-dominated universe as

$$
\epsilon_{a}(t) \equiv \Delta n_{a} /\left[(3 / 2) H^{2}(M / \lambda)\right]=-2 r_{a}^{2} \frac{d \theta_{a}}{d z} .
$$

The lepton number asymmetry is particularly given as the $\tilde{L}$ asymmetry,

$$
\epsilon_{L}(t)=\epsilon_{\tilde{L}}(t)
$$

Since the AD-flaton phases are fluctuating in this early epoch, as mentioned so far, the lepton number asymmetry is oscillating in time as $\left|\epsilon_{L}(t)\right| \sim\left|d \theta_{a} / d z\right| \lesssim\left|\theta_{a}^{(0)}-\theta_{a}^{(1)}\right|$ $\sim 0.01-0.1\left(r_{a} \sim 0.1-1\right)$ numerically for the reasonable parameter values.

$$
\text { C. } m_{3 / 2} \ll H \lesssim H_{\text {osc }}
$$

The high-energy potential terms and the $\bar{h}_{e}$ quartic term are redshifted for $H \gtrsim H_{\text {osc }}$ after the inflation as

$$
V_{\text {high }} \sim H^{3}(M / \lambda),
$$

$$
\bar{h}_{e}^{2}\left|\widetilde{L} H_{d}\right|^{2} \sim \bar{h}_{e}^{2} H^{2}(M / \lambda)^{2},
$$

with $\left|\phi_{a}\right| \sim \sqrt{H(M / \lambda)}$ in Eq. (18). Then, the $\bar{h}_{e}$ quartic term eventually dominates in the present epoch with $H \leqslant H_{\text {osc }}$, playing the crucial role for the flat manifold leptogenesis. Specifically, this quartic term acts in some sense as positive mass-squared terms for the $\widetilde{L}$ and $H_{d}$ fields, driving the ADflaton fields to oscillate. Once the oscillation begins, the lepton number asymmetry is fixed to some nonzero value as

$$
\epsilon_{L}(t)=\epsilon_{L} \quad\left(t \gg H_{\mathrm{osc}}^{-1}\right) .
$$

This asymptotic behavior of $\epsilon_{L}(t)$ in the later time is in accordance with the fact that the lepton number violating terms in $V_{\text {high }}$ are redshifted rapidly to be much smaller than the lepton number conserving terms including the $\bar{h}_{e}$ quartic term. (See Sec. V for the numerical results.) It is also obvious that for $H \gg m_{3 / 2},|\mu|$ the low-energy part $V_{\text {low }}$ still provides negligible effects in this epoch of leptogenesis.

The coherent oscillation of the inflaton field dominates the energy density of the universe until the decay of inflatons is completed at the time $t_{R}\left(\gg H_{\text {osc }}^{-1}\right)$. Then, the universe is reheated to the temperature $T_{R}$. Until this time the lepton number asymmetry is redshifted as matter, which is given at $t=t_{R}\left(H=H_{R}\right)$ with Eqs. (27), (28), and (31) as

$$
n_{L}\left(t_{R}\right)=\epsilon_{L}(3 / 2) H_{R}^{2}(M / \lambda) .
$$

Then, the lepton-to-entropy ratio after the reheating is determined with $s \simeq 3 H_{R}^{2} M_{\mathrm{P}}^{2} / T_{R}$ as 


$$
\begin{aligned}
\frac{n_{L}}{s} & \simeq \epsilon_{L} \frac{(M / \lambda) T_{R}}{2 M_{\mathrm{P}}^{2}} \\
& \sim 10^{-10}\left(\frac{\epsilon_{L}}{0.1}\right)\left(\frac{10^{-6} \mathrm{eV}}{m_{\nu_{1}}}\right)\left(\frac{T_{R}}{10^{9} \mathrm{GeV}}\right),
\end{aligned}
$$

where $M_{\mathrm{P}}=m_{\mathrm{P}} / \sqrt{8 \pi}=2.4 \times 10^{18} \mathrm{GeV}$ is the reduced Planck mass, and the relation (3) between $M / \lambda$ and $m_{\nu_{1}}$ is considered. This lepton number asymmetry is converted partially to the baryon number asymmetry through the electroweak anomalous effect. The chemical equilibrium between leptons and baryons leads the ratio $n_{B}=-(8 / 23) n_{L}$ (without any preexisting baryon number asymmetry) [10]. Therefore the sufficient baryon-to-entropy ratio can be provided as required from the nucleosynthesis with $\eta=(2.6-6.2) \times 10^{-10}$ [11].

It should be noted here that the reheating temperature may be constrained as $T_{R} \lesssim 10^{8}-10^{10} \mathrm{GeV}$, or even more severely, for $m_{3 / 2} \sim 1 \mathrm{TeV}$ to avoid the gravitino problem [1214]. Hence the desired mass of the lightest neutrino is very small generally as $m_{\nu_{1}} \lesssim 10^{-6} \mathrm{eV}$. It will, however, be found in Sec. $\mathrm{V}$ that there is some range of the model parameters for the successful leptogenesis with relatively large $m_{\nu_{1}}$ $\sim 10^{-4} \mathrm{eV}$, if the reheating temperature is allowed to be $T_{R} \sim 10^{10} \mathrm{GeV}$ for $m_{3 / 2} \sim$ several $\mathrm{TeV}$ with a small hadronic branching ratio of gravitino decay $B_{h} \sim 0.01$ [13]. Then, the prediction for the neutrinoless double beta decay with very small $m_{\nu_{1}}$ [5] could be evaded in the case of $\widetilde{L}-H_{u}-H_{d}$ flat manifold leptogenesis.

\section{THERMAL EFFECTS}

We now discuss that the thermal terms for the scalar potential $[4,5,7]$ do not alter essentially the present scenario of flat manifold leptogenesis. Before the reheating after inflation is completed, there is already a dilute plasma of the inflaton decay products with temperature

$$
T_{\mathrm{p}} \sim\left(T_{R}^{2} H M_{\mathrm{P}}\right)^{1 / 4}
$$

Then, the AD-flatons acquire the thermal mass terms in this plasma,

$$
V_{\text {th } 1}=c_{\text {th }} y^{2} T_{\mathrm{p}}^{2}\left|\phi_{a}\right|^{2}\left(y\left|\phi_{a}\right|<T_{\mathrm{p}}\right),
$$

where $y$ is the relevant coupling constant, and $c_{\text {th }}$ is the positive constant, e.g., $c_{\text {th }}=3 / 4$ for a quark superfield. One can readily estimate the Hubble parameter $H_{\text {th1 }}$ when the thermal mass terms begin to dominate over the Hubble induced mass terms:

$$
H_{\mathrm{th} 1} \sim \min \left[\frac{T_{R}^{2} M_{\mathrm{P}}}{y^{4}(M / \lambda)^{2}},\left(y^{4} T_{R}^{2} M_{\mathrm{P}}\right)^{1 / 3}\right] .
$$

The thermal $\log$ terms are also given mainly through the modification of the $\mathrm{SU}(3)_{C}$ gauge coupling as

$$
V_{\mathrm{th} 2}=a_{\mathrm{th}} \alpha_{s}^{2}(T) T_{\mathrm{p}}^{4} \ln \left(\left|\phi_{a}\right|^{2} / T_{\mathrm{p}}^{2}\right)
$$

with $a_{\mathrm{th}} \sim 1$ depending on the particle contents. The Hubble parameter $H_{\text {th2 }}$ for the thermal log terms to be comparable to the Hubble induced mass terms is also estimated with $\left|\phi_{a}\right|$ $\sim \sqrt{H(M / \lambda)}$ as

$$
H_{\mathrm{th} 2} \sim \alpha_{s}\left[T_{R}^{2} M_{\mathrm{P}} /(M / \lambda)\right]^{1 / 2} .
$$

Then, the thermal effects become important for the Hubble parameter

$$
H_{\mathrm{th}} \sim \max \left[H_{\mathrm{th} 1}, H_{\mathrm{th} 2}\right] .
$$

It really takes the maximal value as

$$
\begin{aligned}
H_{\mathrm{th}}^{\max } & \sim H_{\mathrm{th} 2} / \alpha_{s} \\
& \sim 5 \times 10^{8} \mathrm{GeV}\left(\frac{T_{R}}{10^{9} \mathrm{GeV}}\right)\left(\frac{m_{\nu_{1}}}{10^{-6} \mathrm{eV}}\right)^{1 / 2}
\end{aligned}
$$

with a certain value of the relevant coupling,

$$
y \sim 3 \times 10^{-3}\left(\frac{T_{R}}{10^{9} \mathrm{GeV}}\right)^{1 / 4}\left(\frac{m_{\nu_{1}}}{10^{-6} \mathrm{eV}}\right)^{3 / 8},
$$

where Eq. (3) is considered for $m_{\nu_{1}}$ and $M / \lambda$. It is readily seen that $H_{\mathrm{osc}}>H_{\text {th }}$ for a wide range of the model parameters. Then, the leptogenesis is completed dominantly by the $\bar{h}_{e}$ quartic term. On the other hand, one may obtain $H_{\text {th }}$ $\gtrsim H_{\text {osc }}$ for a certain model parameter range with $\bar{h}_{e} \sim 10^{-5}$, $(M / \lambda) \lesssim 10^{18} \mathrm{GeV}$ and $T_{R} \gtrsim 10^{9} \mathrm{GeV}$. Then, the oscillation of AD-flaton fields is driven by the thermal terms rather than the $\bar{h}_{e}$ quartic term. In any case, the lepton number asymmetry fluctuating after the inflation is fixed to some significant nonzero value in the early epoch with $H \gg m_{3 / 2}$. The thermal terms do not severely suppress the lepton number generation, but even play a cooperative role in the flat manifold leptogenesis.

\section{NUMERICAL ANALYSIS}

We here present the results of detailed numerical calculations for the flat manifold leptogenesis. The characteristic features in the multidimensional motion of the AD-flaton fields are confirmed, and the reasonable range of the model parameters is identified for the sufficient leptogenesis.

The values of the various model parameters are taken in the following range:

$$
\begin{aligned}
& M=10^{17} \mathrm{GeV}, \quad H_{\mathrm{inf}}=10^{14} \mathrm{GeV}, \\
& t_{0}=(2 / 3) H_{\mathrm{inf}}^{-1}, \quad \bar{h}_{e}=10^{-5}-10^{-3}, \\
& T_{R}=10^{5} \mathrm{GeV}-10^{10} \mathrm{GeV}, \\
& \lambda_{E}, \lambda_{H}=0.1 \lambda-10 \lambda, \quad \lambda=10^{-3}-1, \\
& \left|a_{t}\right|,\left|a_{H}\right|, c_{a}=0-2,
\end{aligned}
$$


TABLE I. Typical cases for the flat manifold leptogenesis, where $M=10^{17} \mathrm{GeV}$ and $H_{\text {inf }}=10^{14} \mathrm{GeV}$ are taken for definiteness. The index $a$ denotes the AD flatons as $\phi_{a}=\widetilde{L}, H_{u}, H_{d}$ in order.

\begin{tabular}{|c|c|c|c|c|c|c|c|c|c|}
\hline & \multicolumn{3}{|c|}{ Case (1) } & \multicolumn{3}{|c|}{ Case (2) } & \multicolumn{3}{|c|}{ Case (3) } \\
\hline $\bar{h}_{e}$ & \multirow{2}{*}{\multicolumn{3}{|c|}{$\begin{array}{l}3 \times 10^{-5} \\
(10.2,0)\end{array}$}} & \multicolumn{3}{|c|}{$10^{-3}$} & \multicolumn{3}{|c|}{$10^{-3}$} \\
\hline$\left(\tan \beta, \theta_{12}\right)$ & & & & \multicolumn{3}{|c|}{$(2.1, \pi / 4)$} & \multicolumn{3}{|c|}{$(2.1, \pi / 4)$} \\
\hline$\left(M / \lambda, T_{R}\right)$ & \multicolumn{3}{|c|}{ GeV $10^{9}$} & \multicolumn{3}{|c|}{$\left(10^{20} \mathrm{GeV}, 10^{8} \mathrm{GeV}\right)$} & \multicolumn{3}{|c|}{$\left(10^{18} \mathrm{GeV}, 10^{10} \mathrm{GeV}\right)$} \\
\hline$\left(H_{\mathrm{osc}}, H_{\mathrm{th}}^{\max }\right)$ & \multicolumn{3}{|c|}{$\left(9 \times 10^{9} \quad \mathrm{GeV}, 5 \times 10^{8} \mathrm{GeV}\right)$} & \multicolumn{3}{|c|}{$\left(10^{14} \mathrm{GeV}, 10^{7} \mathrm{GeV}\right)$} & \multicolumn{3}{|c|}{$\left(10^{12} \mathrm{GeV}, 10^{10} \mathrm{GeV}\right)$} \\
\hline$\left(\lambda_{E}, \lambda_{H}\right)$ & \multicolumn{3}{|c|}{$\left(1.0 \times 10^{-2}, 1.5 \times 10^{-2}\right)$} & $(1.5 \times 1($ & $0^{-3}, 0.5 \times$ & $\left(10^{-3}\right)$ & \multirow{2}{*}{\multicolumn{3}{|c|}{$\begin{array}{c}\left(4.0 \times 10^{-1}, 1.0 \times 10^{-1}\right) \\
\left(2.0 \mathrm{e}^{i(1 / 3) \pi}, 0.4 \mathrm{e}^{i \pi}\right)\end{array}$}} \\
\hline$\left(a_{E}, a_{H}\right)$ & \multicolumn{3}{|c|}{$\left(1.0 \mathrm{e}^{i(1 / 4) \pi}, 1.0 \mathrm{e}^{i(1 / 2) \pi}\right)$} & \multicolumn{3}{|c|}{$\left(1.5 \mathrm{e}^{-i(1 / 3) \pi}, 0.5 \mathrm{e}^{-i \pi}\right)$} & & & \\
\hline$c_{a}$ & \multicolumn{3}{|c|}{$(1.5,1.0,0.5)$} & \multicolumn{3}{|c|}{$(1.0,1.0,1.0)$} & \multicolumn{3}{|c|}{$(2.0,2.0,0.5)$} \\
\hline$\left(r_{a}^{(0)}\right)$ & 0.738 & 0.753 & 0.148 & 0.397 & 0.954 & 0.868 & 0.313 & 0.640 & 0.558 \\
\hline$\left(\theta_{a}^{(0)}\right)$ & 1.208 & 0 & 3.108 & -1.170 & 0 & 0.237 & -2.040 & 0 & 2.783 \\
\hline$\left(r_{a}^{(1)}\right)$ & 0.775 & 0.823 & 0.277 & 0.392 & 1.047 & 0.971 & 0.282 & 0.711 & 0.654 \\
\hline$\left(\theta_{a}^{(1)}\right)$ & 1.236 & 0.039 & 3.101 & -1.188 & -0.007 & 0.226 & -2.019 & 0.005 & 2.819 \\
\hline $\begin{array}{l}m_{\nu_{1}} \\
\left(\epsilon_{L}, n_{L} / s\right)\end{array}$ & $\begin{array}{r}3 .( \\
(-0.2\end{array}$ & $\begin{array}{l}0 \times 10^{-6} \\
24,-2.1>\end{array}$ & $\begin{array}{l}\mathrm{eV} \\
\left.10^{-10}\right)\end{array}$ & $\begin{array}{r}3.7 \\
(-0.17\end{array}$ & $\begin{array}{l}\times 10^{-7} \mathrm{e} \\
,-1.5 \times 1\end{array}$ & $\begin{array}{l}\mathrm{V} \\
\left.10^{-10}\right)\end{array}$ & $\begin{array}{r}1.0> \\
(-0.11\end{array}$ & $\begin{array}{l}\times 10^{-4} \\
-1.0 \times\end{array}$ & $\begin{array}{l}\mathrm{eV} \\
\left.10^{-10}\right)\end{array}$ \\
\hline
\end{tabular}

and $[0,2 \pi]$ for the phases of coupling parameters. Here, the parameters relevant to the low-energy part $V_{\text {low }}$ is not presented explicitly. It has been checked numerically that the effects of $V_{\text {low }}$ are negligible in the present scenario of flat manifold leptogenesis. While the fixed value of the mass scale $M$ in $W_{\text {non }}$ is presented for definiteness, the results are really obtained as a function of $M / \lambda$. The value of $\tan \beta$ is determined in Eq. (8) for given $\bar{h}_{e}$ with $\theta_{12} \simeq 0$ (small $\nu_{e}-\nu_{\mu}$ mixing) or $\theta_{12} \simeq \pi / 4$ (large $\nu_{e}-\nu_{\mu}$ mixing).

By choosing randomly the model parameter values, we have first determined the initial values of the AD-flaton fields in Eq. (16) just after the inflation. [The initial phase of $H_{u}$ is chosen as $\theta_{H_{u}}^{(0)}=0$ without loss of generality by making a $\mathrm{U}(1)_{Y}$ transformation.] Then, we have solved the equations of motion (17) for a sufficiently long time interval from $t$ $=t_{0}\left(H=H_{\text {inf }}\right)$ to $t \sim 10^{8} t_{0}$, evaluating the particle number asymmetries $\epsilon_{a}(t)$ as functions of time. [In practice, we have solved Eq. (19) for $\chi_{a}$ with Eq. (20) as functions of $z$ $=\ln \left(t / t_{0}\right)$, since the time interval ranges over many orders. The $D$-flat condition (9) is checked to be held within numerical errors.] The dominant contributions to the thermal terms $V_{\text {th } 1}+V_{\text {th2 }}$ are also taken into account for the evolution of the $\mathrm{AD}$-flaton fields. The relevant parameters are taken for the thermal mass terms as $y=m_{q} /\left\langle H_{d}\right\rangle$ and $c_{\text {th }}=3 / 4$ with the $q$ $=d, s$ quarks, $y=m_{c} /\left\langle H_{u}\right\rangle$ and $c_{\text {th }}=3 / 4$ with the $c$ quark, and $y=m_{\mu} /\left\langle H_{d}\right\rangle$ and $c_{\text {th }}=1 / 4$ with the muon. These Yukawa couplings may be close to the optimal value as given in Eq. (41), depending on $\tan \beta$. As for the thermal $\log$ term, $a_{\mathrm{th}}$ $=9 / 8$ is taken due to the decoupling of the top quark from the thermal plasma.

We have actually made calculations by taking hundreds of the parameter sets. Some typical cases are listed in Table I, where the sufficient lepton number asymmetry is obtained. Cases (1) and (2) correspond to the small $\bar{h}_{e}$ (small $\nu_{e}-\nu_{\mu}$ mixing) and the large $\bar{h}_{e}$ (large $\nu_{e}-\nu_{\mu}$ mixing), respectively. Although different values are taken for $M / \lambda$ and $T_{R}$ in cases (1) and (2), the resultant $\epsilon_{L}$ has only moderate dependence on these parameters. It should be noticed here that $H_{\text {osc }}$ $\sim H_{\text {inf }}$ in case (2). Then, the $\bar{h}_{e}$ quartic term as well as the high-energy part $V_{\text {high }}$ may be important to determine the potential minimum in the inflation epoch. We have really confirmed that even in such an extreme case the multidimensional motion of the $\mathrm{AD}$ fields can be realized producing the significant lepton number asymmetry.

We have also searched the specific parameter range allowing for somewhat high $T_{R} \sim 10^{10} \mathrm{GeV}$, where the sufficient lepton number asymmetry is obtained with relatively large $m_{\nu_{1}} \sim 10^{-4} \mathrm{eV}$ for the lightest neutrino. A typical example is presented as case (3) in Table I.

The time evolution of the AD-flaton fields has been determined precisely by these numerical calculations. In Fig. 1, the multidimensional motion of the AD-flaton fields is typically depicted in terms of the dimensionless fields $\chi_{a}$ for case (1) in Table I. In Fig. 2 is also shown the time variation of the particle number asymmetries, or the contributions to the hypercharge asymmetry, $\epsilon_{\tilde{L}}$ (bold line), $-\epsilon_{H_{u}}$ (slim line), and $\epsilon_{H_{d}}$ (dashed line), which are evaluated with the solutions of $\chi_{a}$. Here, we can check the hypercharge conservation (within the numerical errors), $\epsilon_{\tilde{L}}-\epsilon_{H_{u}}+\epsilon_{H_{d}}=0$. The lepton number asymmetry is given just by $\epsilon_{L}=\epsilon_{\tilde{L}}$. These asymmetries are really fluctuating after the inflation, and then fixed to certain values for $t \gg H_{\mathrm{osc}}^{-1}$.

As for the thermal effects, we have observed that they do not essentially alter the present scenario of flat manifold leptogenesis. In a wide range of the model parameter space, the thermal effects on the final lepton number asymmetry $\epsilon_{L}$ are found to be small for $H_{\mathrm{osc}} \gg H_{\text {th }}$, particularly with lower reheating temperature $T_{R} \lesssim 10^{8} \mathrm{GeV}$. It should, however, be noted that the lepton number asymmetry varies slowly with $z=\ln \left(t / t_{0}\right)$, as seen in Fig. 2. Then, it actually takes a rather long term ranging over some orders around $t \sim H_{\text {osc }}^{-1}$ to completely fix the lepton number asymmetry by driving the ADflaton oscillation. In such a situation, when $H_{\mathrm{th}}^{\max }$ is smaller 

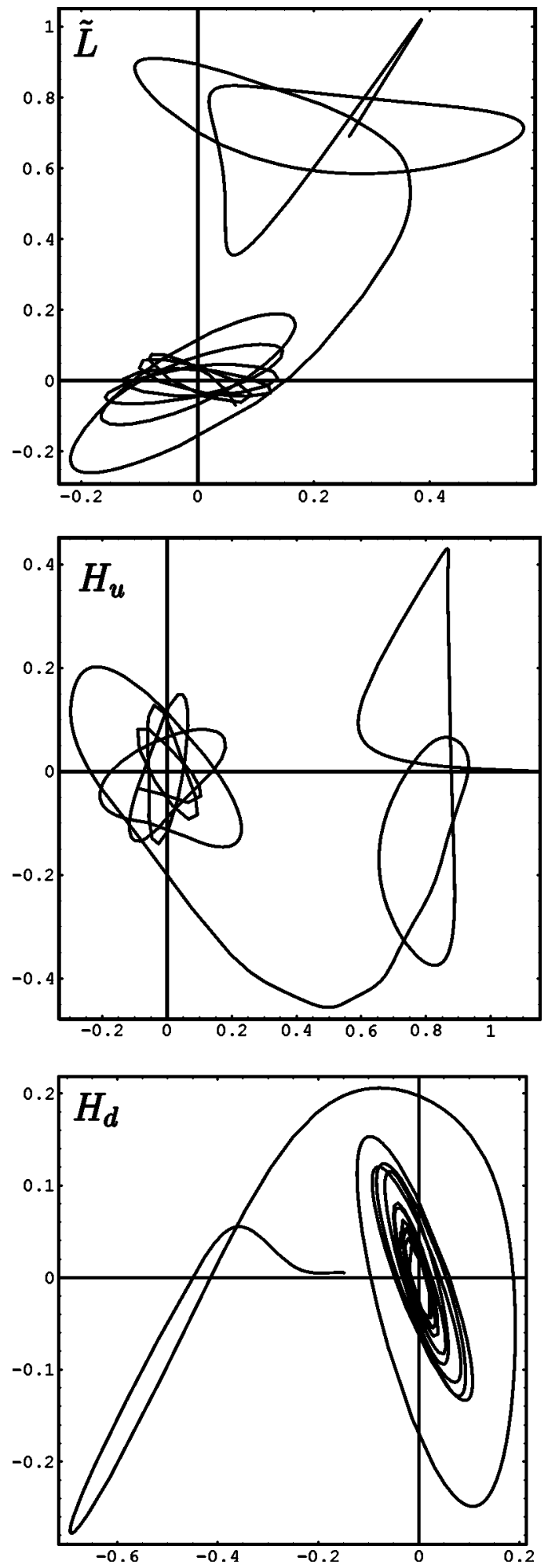

FIG. 1. The motions of the AD-flaton fields, the real part (horizontal axis) and imaginary part (vertical axis), are depicted in terms of the dimensionless fields $\chi_{a}$ for case (1) in Table I.

only by a few orders than $H_{\text {osc }}$, as seen for case (1) of Table I, the thermal terms become dominant at the late stage of leptogenesis for driving the AD-flaton oscillation. In Fig. 3, the time variation of the lepton number asymmetry $\epsilon_{L}(t)$ is shown for case (1) in Table I, where the solid and dashed

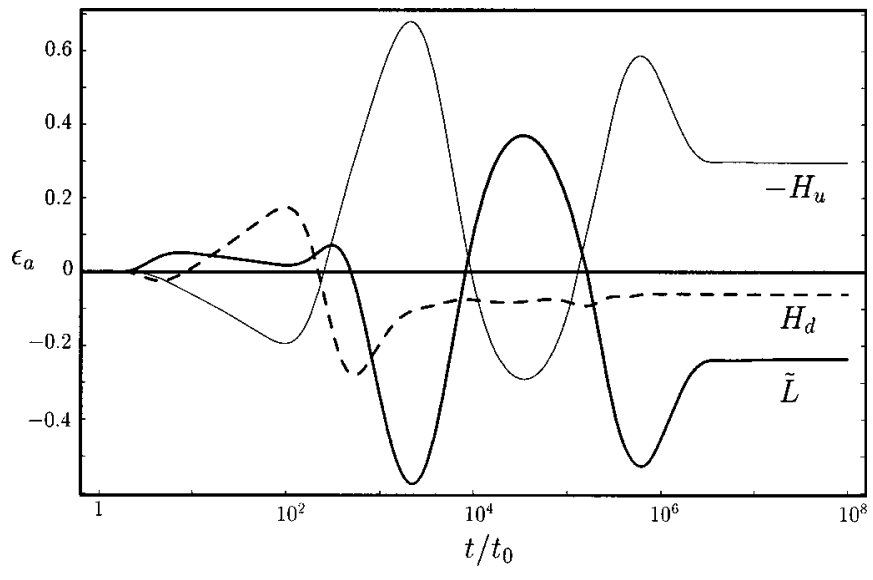

FIG. 2. The time variation of particle number asymmetries is shown for case (1) in Table I.

lines represent the results with and without the thermal terms, respectively. In this case, the thermal log term is considered to provide the dominant effect. We really observe here that the lepton number asymmetry is finally fixed by the thermal terms, though the $\bar{h}_{e}$ quartic term first triggers the AD-flaton oscillation. The resultant $\epsilon_{L}$ is changed by some factor $\sim 1$ due to the thermal effects. We may even have $H_{\mathrm{osc}} \lesssim H_{\mathrm{th}}^{\max }$ for some cases with smaller $\bar{h}_{e} \sim 10^{-5}, M / \lambda$ $\lesssim 10^{18} \mathrm{GeV}$, and higher $T_{R} \gtrsim 10^{9} \mathrm{GeV}$. Then, the AD-flaton oscillation to complete the leptogenesis is driven mainly by the thermal terms rather than the $\bar{h}_{e}$ quartic term. In any case, the thermal terms do not provide severe suppression, but may even play a cooperative role for the flat manifold leptogenesis.

The magnitudes of AD-flaton fields are found to be scaled roughly as

$$
\left|\phi_{a}\right| \propto H^{\alpha(t)}
$$

In Fig. 4, this redshift is shown typically for case (1) of Table I in terms of the dimensionless variables $r_{a}(t) \propto H^{\alpha(t)-1 / 2}$. It

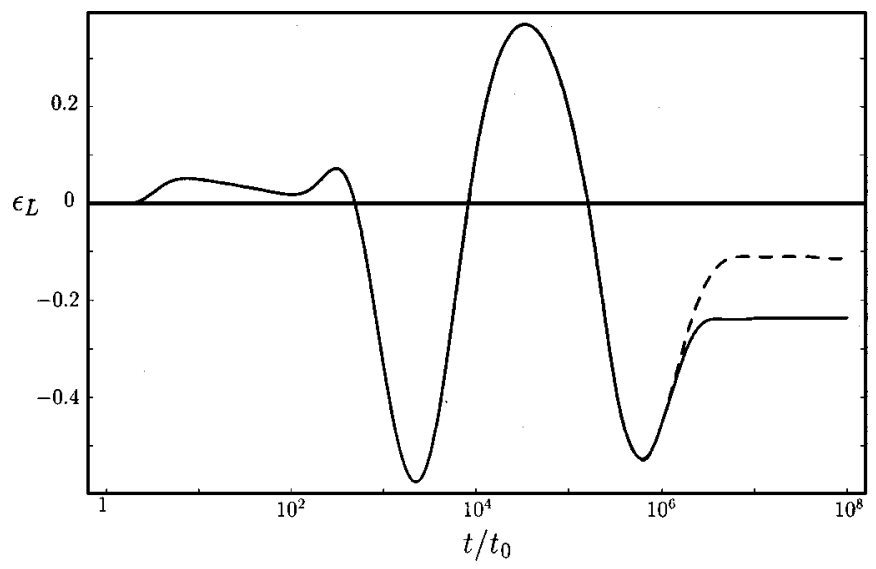

FIG. 3. The time variation of the lepton number asymmetry is shown for case (1) in Table I, where the solid and dashed lines represent the results with and without the thermal terms, respectively. 


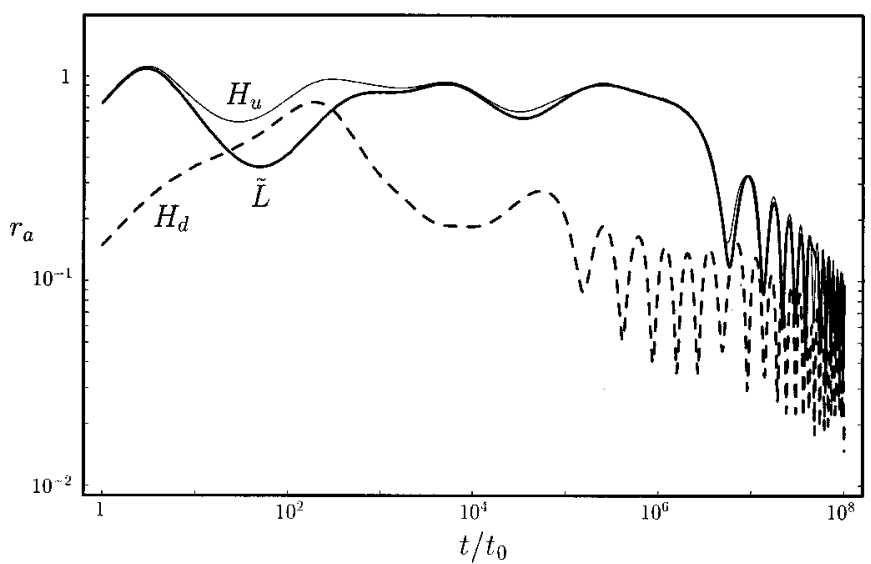

FIG. 4. The time variation of the AD-flaton field magnitudes is shown in terms of $r_{a}$ for case (1) in Table I.

is observed up to some fluctuating behavior that the power of redshift $\alpha(t)$ eventually changes as

$$
\alpha(t) \approx \begin{cases}1 / 2 & \left(t<H_{\mathrm{osc}}^{-1}\right), \\ 2 / 3 & \left(H_{\mathrm{osc}}^{-1} \lesssim t<H_{\mathrm{th}}^{-1}\right), \\ 7 / 8 & \left(H_{\mathrm{th}}^{-1} \lesssim t<m_{3 / 2}^{-1}\right), \\ 1 & \left(t \gtrsim m_{3 / 2}^{-1}\right) .\end{cases}
$$

We here find especially that in the late epoch $H_{\mathrm{th}}^{-1} \lesssim t$ $<m_{3 / 2}^{-1}$ the evolution of the AD-flatons is determined dominantly by the thermal terms, while the leptogenesis is already completed during the epoch $H_{\mathrm{osc}}^{-1} \lesssim t \lesssim H_{\mathrm{th}}^{-1}$. The redshift of the AD-flaton fields changes finally to $\left|\phi_{a}\right| \propto H$, when the low-energy soft supersymmetry breaking mass terms become dominating.

In Fig. 5, the time variation of the scalar potential terms is also shown in terms of the dimensionless effective potential $U\left(\chi_{a}\right)$ in Eq. (21), where the symbols indicate the respective terms as $[c]$ : Hubble induced negative mass-squared terms, $[a]$ : Hubble induced $A$ terms, $[F]:|F|^{2}$ terms from $W_{\text {non }}$,

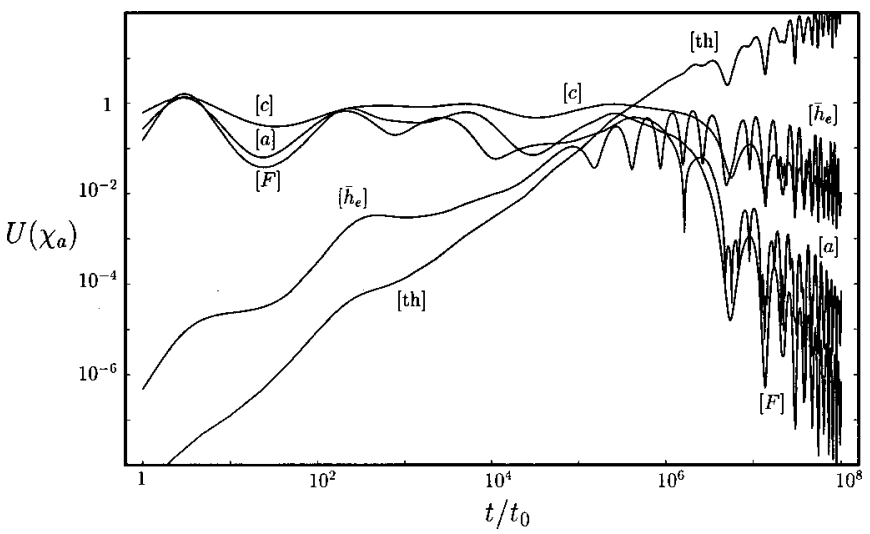

FIG. 5. The time variation of the scalar potential terms is shown in terms of $U(\chi)$ for case (1) in Table I. The respective terms are indicated as $[c]$ : Hubble induced negative mass-squared terms, $[a]$ : Hubble induced $A$ terms, $[F]:|F|^{2}$ terms from $W_{\text {non }},\left[\bar{h}_{e}\right]:\left|\widetilde{L} H_{d}\right|^{2}$ term, and [th]: thermal terms.

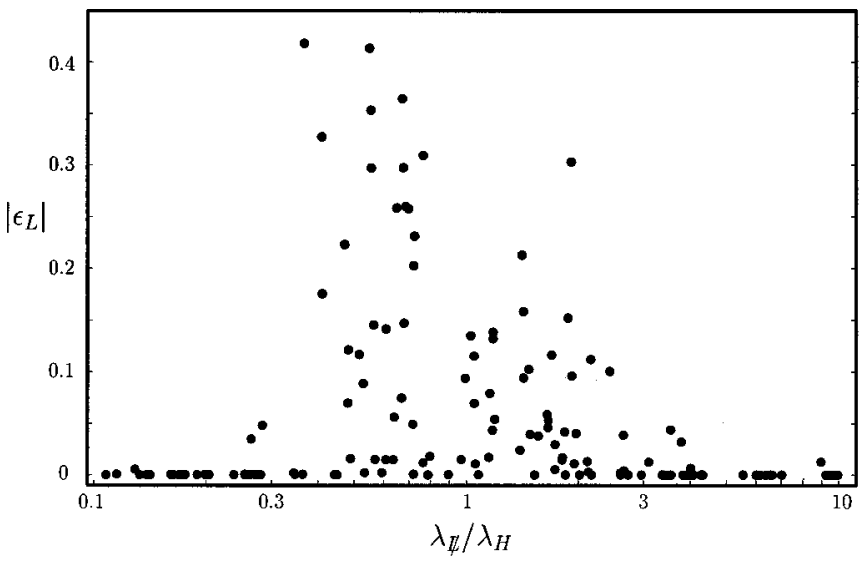

FIG. 6. A scatter plot is presented for the resultant lepton number asymmetries depending on the ratio $\lambda_{E} / \lambda_{H}$, where the relevant parameter values are taken randomly.

$\left[\bar{h}_{e}\right]:\left|\widetilde{L} H_{d}\right|^{2}$ term, and [th]: thermal terms. It is observed that in the epoch with $H>H_{\mathrm{osc}}$ the $[c],[a]$, and $[F]$ terms in $V_{\text {high }}$ really dominate being scaled as $H^{0}$ in terms of the $U\left(\chi_{a}\right)$. Then, the $\left[\bar{h}_{e}\right]$ and even [th] terms catch up to them around $H \sim H_{\mathrm{osc}}$. Soon after that, the [th] term dominates over the $\left[\bar{h}_{e}\right]$ and $[c]$ terms, and the $[a]$ and $[F]$ terms including the lepton number violation decrease rapidly, so that the lepton number asymmetry is fixed to some nonzero value. The $D^{2}$ term is not shown here for simplicity. Since $D$ $=\left(g^{2}+g^{\prime 2}\right)^{1 / 2}\left(|\widetilde{L}|^{2}-\left|H_{u}\right|^{2}+\left|H_{d}\right|^{2}\right)$ is calculated by using the solutions of $\phi_{a}(t)$, it is very sensitive to the numerical errors for the cancellation among $\left|\phi_{a}\right|^{2}$ terms by several orders. We have really checked that the $D^{2}$ contribution is smaller than $10^{-3}$ in the unit of $U\left(\chi_{a}\right)$, though it apparently exhibits a violent oscillation within this small range. This oscillating behavior is regarded to be an artifact within the intrinsic numerical errors due to the fine cancellation among the large $\left|\phi_{a}\right|^{2}$ terms in calculating the $D$ term. The $D^{2}$ term is anyway small enough compared to the leading terms in the $U\left(\chi_{a}\right)$, and the $D$-flat condition (9) is maintained quite well through the evolution of the AD-flaton fields.

It has been argued that the comparability of $\lambda_{t}$ and $\lambda_{H}$ is essential for the flat manifold leptogenesis. In Fig. 6, the resultant lepton number asymmetries are plotted depending on the ratio $\lambda_{E} / \lambda_{H}$, where the relevant parameter values are taken randomly. It is clearly seen that the flat manifold leptogenesis can be realized naturally under the flatness condition (10).

\section{CONCLUSION}

We have investigated the flat manifold leptogenesis in the manner of Affleck and Dine with the slepton and Higgs fields, $\widetilde{L}, H_{u}, H_{d}$, in the supersymmetric standard model. The multidimensional motion of these AD-flaton fields is indeed realized in the case that the $\widetilde{L} H_{u}$ and $H_{u} H_{d}$ directions are comparably flat with the relevant nonrenormalizable superpotential terms. Soon after inflation, the lepton number asymmetry appears to fluctuate due to this multidimensional 
motion involving certain $C P$ violating phases. Then, the lepton number asymmetry is fixed to some significant nonzero value for the successful baryogenesis when the scalar fields begin to oscillate with rotating phases driven by the quartic coupling from the superpotential term $\bar{h}_{e} L H_{d} e^{c}$ with $\bar{h}_{e}$ $\sim 10^{-5}-10^{-3}$. The Hubble parameter $H_{\text {osc }}$ at this epoch for the completion of leptogenesis is much larger than the gravitino mass $m_{3 / 2} \sim 10^{3} \mathrm{GeV}$. The thermal terms do not alter this scenario of flat manifold leptogenesis in the early epoch. They may even play a cooperative role for leptogenesis. The lightest neutrino mass can be $m_{\nu_{1}} \sim 10^{-4} \mathrm{eV}$ if the reheating temperature is allowed to be $T_{R} \sim 10^{10} \mathrm{GeV}$. Clearly, this flat manifold leptogenesis is not restricted by the physics at the electroweak scale such as the low-energy supersymmetry breaking terms.

\section{ACKNOWLEDGMENTS}

This work was supported in part by Grant-in-Aid for Scientific Research on Priority Areas B (No. 13135214) from the Ministry of Education, Culture, Sports, Science and Technology, Japan.
[1] I. Affleck and M. Dine, Nucl. Phys. B249, 361 (1985).

[2] M. Dine, L. Randall, and S. Thomas, Nucl. Phys. B458, 291 (1996).

[3] H. Murayama and T. Yanagida, Phys. Lett. B 322, 349 (1994); T. Moroi and H. Murayama, J. High Energy Phys. 07, 009 (2000); M. Fujii, K. Hamaguchi, and T. Yanagida, Phys. Rev. D 65, 043511 (2002).

[4] T. Asaka, M. Fujii, K. Hamaguchi, and T. Yanagida, Phys. Rev. D 62, 123514 (2000).

[5] M. Fujii, K. Hamaguchi, and T. Yanagida, Phys. Rev. D 63, 123513 (2001); Phys. Lett. B 538, 107 (2002).

[6] M. Senami and K. Yamamoto, Phys. Lett. B 524, 332 (2002).

[7] R. Allahverdi, B.A. Campbell, and J. Ellis, Nucl. Phys. B579, 355 (2000); A. Anisimov and M. Dine, ibid. B619, 729 (2001).

[8] T. Yanagida, in Proceedings of Workshop on Unified Theory and Baryon Number in the Universe, edited by O. Sawada and A. Sugamoto (KEK, Tsukuba, 1979); M. Gell-Mann, P. Ramond, and R. Slansky, in Supergravity, edited by P. van Nie- wenhuizen and D.Z. Freedman (North-Holland, Amsterdam, 1979).

[9] K. Yamamoto, Phys. Lett. 161B, 289 (1985); 168B, 341 (1986).

[10] J.A. Harvey and M.S. Turner, Phys. Rev. D 42, 3344 (1990).

[11] Particle Data Group, D.E. Groom et al., Eur. Phys. J. C 15, 1 (2000), http://pdg.lbl.gov/.

[12] J. Ellis, J.E. Kim, and D.V. Nanopoulous, Phys. Lett. 145B, 181 (1984); E. Holtmann, M. Kawasaki, K. Kohri, and T. Moroi, Phys. Rev. D 60, 023506 (1999); M. Kawasaki, K. Kohri, and T. Moroi, ibid. 63, 103502 (2001).

[13] K. Kohri, Phys. Rev. D 64, 043515 (2001).

[14] R. Kallosh, L. Kofman, A. Linde, and A.V. Proeyen, Phys. Rev. D 61, 103503 (2000); G.F. Giudice, I. Tkachev, and A. Riotto, J. High Energy Phys. 08, 009 (1999); 11, 036 (1999); H.P. Nilles, M. Peloso, and L. Sorbo, Phys. Rev. Lett. 87, 051302 (2001). 